\title{
Ultrafast photofragmentation dynamics of molecular iodine driven with timed XUV and near-infrared light pulses
}

\author{
Maria Krikunova, ${ }^{\text {a) }}$ Theophilos Maltezopoulos, Philipp Wessels, Moritz Schlie, \\ Armin Azima, Marek Wieland, and Markus Drescher \\ Institut für Experimentalphysik, Universität Hamburg, Luruper Chaussee 149, 22761 Hamburg, Germany
}

(Received 27 July 2010; accepted 30 November 2010; published online 12 January 2011)

\begin{abstract}
Photofragmentation dynamics of molecular iodine was studied as a response to the joint illumination with femtosecond $800 \mathrm{~nm}$ near-infrared and $13 \mathrm{~nm}$ extreme ultraviolet (XUV) pulses delivered by the free-electron laser facility FLASH. The interaction of the molecular target with two light pulses of different wavelengths but comparable pulse energy elucidates a complex intertwined electronic and nuclear dynamics. To follow distinct pathways out of a multitude of reaction channels, the recoil of created ionic fragments is analyzed. The delayed XUV pulse provides a way of following molecular photodissociation of $I_{2}$ with a characteristic time-constant of $(55 \pm 10)$ fs after the laser-induced formation of antibonding states. A preceding XUV pulse, on the other hand, preferably creates a $4 d^{-1}$ inner-shell vacancy followed by the fast Auger cascade with a revealed characteristic time constant $\tau_{\mathrm{A} 2}=(23 \pm 11)$ fs for the second Auger decay transition. Some fraction of molecular cationic states undergoes subsequent Coulomb explosion, and the evolution of the launched molecular wave packet on the repulsive Coulomb potential was accessed by the laser-induced postionization. A further unexpected photofragmentation channel, which relies on the collective action of XUV and laser fields, is attributed to a laser-promoted charge transfer transition in the exploding molecule. (C) 2011 American Institute of Physics. [doi:10.1063/1.3528722]
\end{abstract}

\section{INTRODUCTION}

Illumination of a molecule with visible light or with extreme ultraviolet (XUV) radiation leading to electronic or nuclear excitation and relaxation may both destroy a chemical bond; the involved processes and the explicit dynamics, however, will usually be quite different. Excitation with ultraviolet, visible, or near-infrared (NIR) laser radiation will act only on the least bound valence electrons, even in multiphoton processes at high intensities. XUV photons, as routinely delivered from synchrotron storage ring facilities, usually favor excitation of inner electronic shells; the large deposited energy leads to rapid electronic de-excitation, e.g., via Auger decay. However, both excitation modes may form highly excited or multiply charged molecular ions finally leading to molecular dissociation or Coulomb explosion.

While both wavelength regimes were investigated independently in the past, novel light sources such as laserbased high-harmonic generation (HHG) or accelerator-based free-electron-laser (FEL) now deliver synchronized ultrashort light pulses in the XUV as well as in the visible range. This has allowed the realization of visible-pump/XUV-probe ${ }^{1-3}$ or XUV-pump/NIR-probe ${ }^{4,5}$ experiments, revealing ultrafast nuclear and electronic dynamics, respectively. A very recent study ${ }^{6}$ probes visible-driven photodissociation of $\mathrm{Br}_{2}$ by spectrally analyzing high-harmonic radiation formed with a

\footnotetext{
a) Author to whom correspondence should be addressed. Present address: Institut für Optik und Atomare Physik, Technische Universität Berlin, Hardenbergstr. 36, 10623 Berlin, Germany. Electronic mail: maria.krikunova@physik.tu-berlin.de.
}

delayed laser pulse. This technique, however, currently does not include the excitation with XUV.

The comparable intensity provided in a synchronized way by FEL facilities for both wavelengths, on the other hand, facilitates a more general concept beyond pump-probe, where the evolution is governed by the collective action of the two light fields, each driving dynamical processes with similar probability but in a very different manner. In contrast to the visible range, where the molecular photodissociation can be started from the transient state of a specific configuration, the inner-shell core-ionization produces a multitude of molecular cationic states giving rise to different fragmentation pathways. For molecules such as $\mathrm{HBr}, \mathrm{CH}_{3} \mathrm{Br}$, and $\mathrm{HI}$, the XUV-induced photofragmentation has been shown to be competitive with the decay of XUV-created core holes. ${ }^{7}$ For molecules built of heavy atoms such as $\mathrm{I}_{2}$, however, the electronic relaxation is expected to precede photodissociation. In this work, we are utilizing femtosecond XUV pulses from the FEL in Hamburg FLASH in combination with synchronized NIR laser pulses for studying their mutual impact on iodine molecules.

The sensitivity of photoelectron spectroscopy to a specific electronic configuration makes this detection method a powerful approach in studies of molecular photodissociation. In an energy-domain study on the XUV-induced core excitation of $\mathrm{HBr}^{8}{ }^{8}$ a two-step relaxation process involving a fast neutral dissociation $\mathrm{H}+\mathrm{Br}^{*}$ followed by the autoionization of an excited fragment has been identified. Time-resolved studies on the laser-induced photodissociation of $\mathrm{Br}_{2}$ (Refs. 1-3) have revealed the transition from the bound molecule to two distinct atoms within less than 
100 fs using pulsed XUV photoelectron spectroscopy of the valence states. According to the authors experience with the application of photoelectron spectroscopy at FEL sources, ${ }^{9}$ this method is subject to several challenges: (i) the typical bandwidth of more than $1 \mathrm{eV}$ at $90 \mathrm{eV}$ photon energy delivered by FLASH prohibits the observation of subtle changes in the photoelectron spectra, while spectral filtering with the available monochromator ${ }^{10}$ compromises the temporal resolution; (ii) the electron spectrum is further complicated by the formation of sidebands from absorption of several NIR photons $;{ }^{11}$ and (iii) the spectrum of relevant Auger electrons originating from the second step of an Auger cascade is too dense to reveal any spectral feature.

In this work, we observe the kinetic energy release (KER) of fragments resulting from the recoil of the Coulomb explosion of a multiply charged molecular ion. For molecules this observable delivers more comprehensive information than the mere detection of the ion charge states ${ }^{4,5}$ and avoids the complications (i)-(iii). Generally, ions with higher charge states and higher KER are produced by a reduced number of possible pathways, thereby increasing the selectivity of the method and the expressiveness of the results. The interpretation in Sec. III, therefore, mainly focuses on the charge states $\geq 2$ and $\mathrm{KER}>6 \mathrm{eV}$.

\section{EXPERIMENT}

The experiment was performed at beamline BL1 of the FLASH facility operated at a photon energy near $92 \mathrm{eV}$ (13.4 $\mathrm{nm}$ wavelength) and a $5 \mathrm{~Hz}$ repetition rate in single bunch mode. ${ }^{12} \mathrm{~A}$ mode-locked Ti:sapphire laser system [800 nm, 120 fs full-width-at-half-maximum (FWHM) pulse duration, pulse energy $2 \mathrm{~mJ}$, provided by the FLASH facility, is electronically synchronized to the $1.3 \mathrm{GHz}$ master clock of the accelerator. In the interaction region of the experiment the $800 \mathrm{~nm}$ NIR laser beam of $60 \mu \mathrm{m}$ FWHM spot size was overlapped with the XUV pulse of about $150 \mu \mathrm{m}$ FWHM spot size at an angle of $\sim 2^{\circ}$, thus keeping a blurring of the temporal overlap between both pulses due to the noncollinear geometry below $17 \mathrm{fs}$.

Molecular iodine was expanded into the interaction region of the pump-probe chamber with a background pressure of $\sim 10^{-7}$ mbar through a slightly heated gas nozzle and recollected with a liquid nitrogen cold trap. The chamber pressure with operating gas nozzle was kept in the range of $10^{-6}$ mbar. Momentum resolved charge states of $\mathrm{I}^{p+}$ ions created in the target volume were detected by a time-of-flight (TOF) spectrometer. It consists of an extraction and an acceleration region with electrical field strengths of 25 and 500 $\mathrm{V} / \mathrm{mm}$, respectively, attached to a $62 \mathrm{~cm}$ long field free flight tube. A $2 \mathrm{~mm}$ diameter aperture at the exit of the acceleration region was used to restrict the detection solid angle. The polarization vectors of XUV and NIR laser beams were directed parallel to the detector axis.

Figure 1 shows the momentum resolved $\mathrm{I}^{+}$(a) and $\mathrm{I}^{2+}$ (b) fragment ion spectra induced by exclusive irradiation with the XUV field (solid line). As known from synchrotron radiation studies, the most prominent feature in the XUV absorption spectrum of $I_{2}$ is a strong continuum peak with its maximum near $93 \mathrm{eV}$ (Ref. 13) interpreted as a $4 d$ $\rightarrow \varepsilon f$ shape resonance in close similarity to xenon. ${ }^{14}$ The $4 d^{-1}$ vacancy created by the XUV radiation in one of the iodine atoms decays via a single (A1) or a cascade of two Auger processes $(\mathrm{A} 1+\mathrm{A} 2)$, creating doubly $\mathrm{I}_{2}^{2+}$ (A1) or triply $\mathrm{I}_{2}^{3+}$ (A1+A2) charged molecular states, respectively. These transient molecular ionic states have been shown to dissociate further via $\mathrm{I}^{+}+\mathrm{I}^{+}$and $\mathrm{I}^{2+}+\mathrm{I}^{+}$fragmentation pathways. ${ }^{15}$ If the photofragmentation is governed by repulsive forces, the corresponding fragments will possess a characteristic KER of $(1,1)$ and $(1,2) /(2,1)$, respectively.

In this nomenclature, the photofragmentation channels governed by the Coulomb explosion $\mathrm{I}_{2}^{p+q} \rightarrow \mathrm{I}^{p+}+\mathrm{I}^{q+}$ are referred to as $(p, q)$, with the first number $p$ corresponding to the charge of the detected ion. The Coulomb potential of each $(p, q)$ channel corresponds to the total KER of charged atomic fragments and is defined by $p \cdot q / R_{e}$ (in atomic units), where $R_{e}$ is the equilibrium internuclear distance of the neutral molecule $\left(R_{e}=2.66 \AA\right.$ for $\left.\mathrm{I}_{2}\right)$. Fragments from each $(p, q)$ channel exhibit two peaks in the ion yield spectra, one with shorter and one with longer TOF corresponding, respectively, to ions initially flying forward (fw) and backward (bw) with respect to the detector. The TOF of each $(p, q)$ fragmentation channel was identified by a simulation of the ion trajectories with Simion 8.0. In Fig. 1(a), the $(1,1)$ and $(1,2)$ fragmentation channels are superimposed, while the $(2,1)$ channel appears as a pronounced maximum in Fig. 1(b). With an average XUV target intensity in the order of $8 \times 10^{11} \mathrm{~W} / \mathrm{cm}^{2}$, as it can be estimated by assuming a 35 fs FWHM pulse duration, ${ }^{16}$ the contribution of XUV-induced multiphoton processes leading to a sum charge $p+q>3$ is considered to be negligible. This is corroborated by the absence of ionic fragments with a charge state higher than $\mathrm{I}^{2+}$ produced solely by the XUV radiation.

Figure 1(a) exhibits a pronounced maximum at zero KER where the detection efficiency of the TOF spectrometer is highest, dropping significantly with rising fragment momentum. As it is corroborated by the analysis of the time-resolved spectra (see Sec. III B), the majority of the signal for the zero KER feature is probably caused by a molecular dication $\mathrm{I}_{2}^{2+*}$ trapped in a bound state. It will appear at the same TOF corresponding to the same mass-to-charge ratio as $\mathrm{I}^{+}$and will have no KER. The $\mathrm{I}_{2}^{3+}$ and $\mathrm{I}_{2}^{+}$signals were also resolved in our TOF spectra (data not shown), but the amplitude of both was noticeably smaller.

Care was taken to avoid strong field ionization of the molecular ground state such as multielectron dissociative ionization (MEDI). ${ }^{17,18}$ At NIR intensities above $5 \times 10^{13} \mathrm{~W} / \mathrm{cm}^{2}, \mathrm{I}_{2}$ was shown ${ }^{18}$ to loose several electrons and to undergo Coulomb explosion. Thus, by keeping the NIR intensity below $10^{13} \mathrm{~W} / \mathrm{cm}^{2}$, the $(p, q)$ fragmentation channels can unambiguously be attributed to the action of the XUV field.

Under the current experimental conditions the laserinduced photodissociation most probably proceeds via the repulsive $\mathrm{B}{ }^{1} \Pi_{u}^{+}$as well as via the bound $\mathrm{B}{ }^{3} \Pi_{u}^{+}$state, excited by multiphoton absorption. The photodissociation from these states predominantly leads to the production of iodine atoms (I) in the ${ }^{2} P_{3 / 2}$ ground state. ${ }^{19}$ However, the production of atoms in an excited ${ }^{2} P_{1 / 2}$ state above the $\mathrm{B}$ state 
(a)

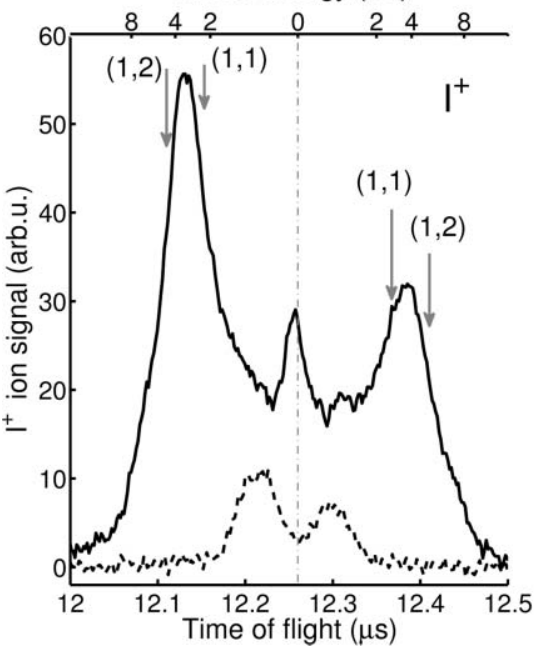

(b)

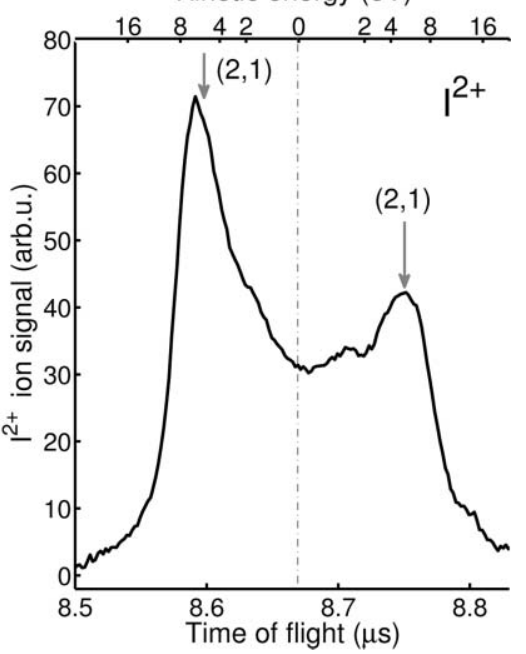

FIG. 1. Momentum resolved $\mathrm{I}^{+}$(a) and $\mathrm{I}^{2+}$ (b) ion yield spectra produced exclusively by XUV (solid line) and NIR (dashed line) fields. Measured data were typically averaged over 80 single traces. The scale at the top indicates the fragment kinetic energy (half of KER) for ions with the initial momentum on the spectrometer's axis. The corresponding $(p, q)$ channels are indicated by vertical arrows. The dash-dotted line denotes zero KER. Note that only $\mathrm{I}^{+}$fragment ions with low KER are produced by the NIR field under the current experimental conditions.

dissociation limit cannot completely be excluded. Theoretical considerations predict that higher energy valence shell states have an ion-pair structure, i.e., $\mathrm{I}^{-}+\mathrm{I}^{+}$, however, these states shortcut to a dissociative limit of $\mathrm{I}+\mathrm{I}^{*} \cdot{ }^{20}$ Figure 1(a) (dashed line) shows that only a small fraction of $\mathrm{I}$ and $\mathrm{I}^{*}$ is further ionized within the same NIR pulse by a postdissociative ionization (PDI) process. The KER of these fragments is considerably lower as compared to Coulomb repulsion.

A methodological challenge for pump-probe experiments employing self-amplified spontaneous emission (SASE) FELs is connected with arrival time fluctuations introducing a timing jitter in the order of a few hundreds of femtoseconds between XUV and NIR laser pulses. ${ }^{9,11}$ Without specially developed tools ${ }^{21}$ this inevitable timing jitter will significantly limit the temporal resolution of a pump-probe experiment. In a previous work we introduced a single-shot XUV/NIR crosscorrelator $^{22}$ for a simultaneous arrival time measurement of the XUV with respect to the NIR pulse. After an experimental run the pump-probe data can be rearranged according to this information. As it was demonstrated previously ${ }^{23}$ by applying this tagging concept, the XUV timing jitter can be compensated down to the level where the NIR pulse duration becomes the dominating parameter in delimiting the temporal resolution. The zero time delay, defined as a coincidence of the XUV and NIR maxima, was determined by analyzing the temporal profile arising from the Coulomb explosion of the $\mathrm{I}_{2}^{3+} \rightarrow(2,1)$ molecular cationic state, as discussed in Sec. III B. The temporal width $\sigma=(53 \pm 10)$ fs extracted from the data analysis is comparable with the pulse duration of the optical laser of $\sigma_{\text {laser }}=50 \mathrm{fs}$ (120 fs FWHM) and thus indicates an effective jitter compensation in the current study.

\section{RESULTS AND DISCUSSION}

The momentum resolved transient $\mathrm{I}^{+}, \mathrm{I}^{2+}$, and $\mathrm{I}^{3+}$ ion yields as a function of the time delay between XUV and NIR pulses are presented in Fig. 2. The KER data were collected at a fixed nominal optical delay stage position by a simultaneous measurement of the XUV arrival time as described in Ref. 23. Each ion trace represents an average of about 200 single traces within 30 fs time bins. The time resolved spectra in Fig. 2 contain very rich information. The detailed

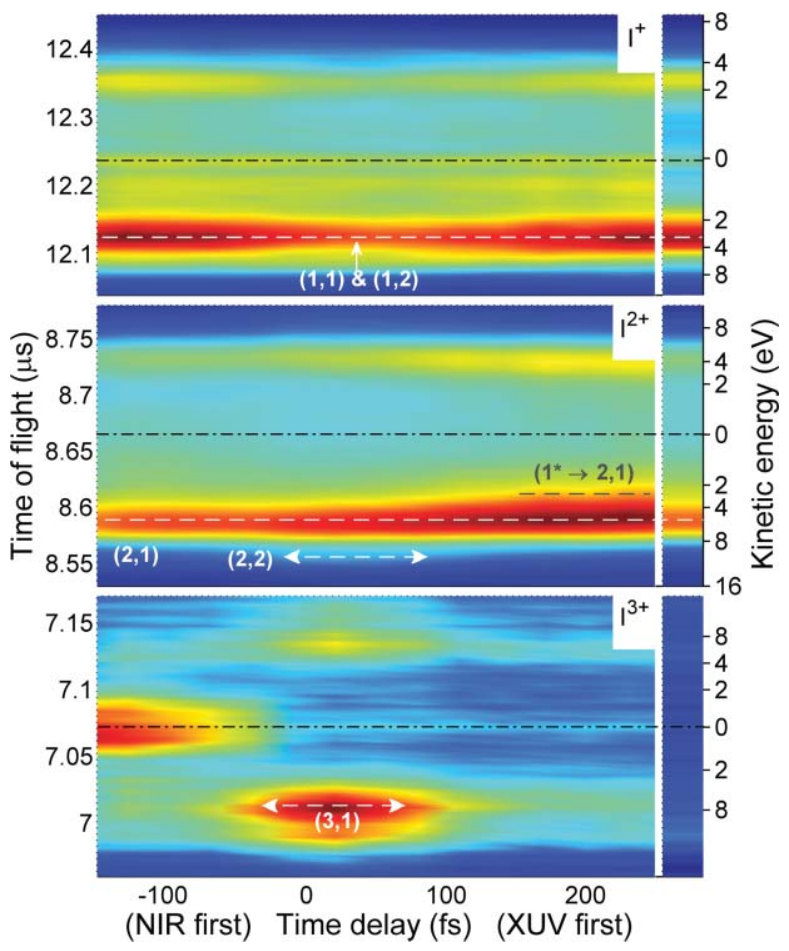

FIG. 2. Momentum resolved transient $\mathrm{I}^{+}, \mathrm{I}^{2+}$, and $\mathrm{I}^{3+}$ ion yields as a function of the time delay between XUV and NIR pulses. The data were sorted according to the XUV arrival time measured simultaneously with an XUV/NIR cross-correlator and averaged subsequently within $30 \mathrm{fs}$ time bins. Each ion trace was averaged over about 200 single traces. The axis at the right indicates the fragment kinetic energy (half of KER) for ions with the initial momentum on the spectrometer's axis. The decay channels under study are labeled with $(p, q)$. Plotted to the right is the corresponding ion yield solely induced by the XUV pulse. 
Process A: NIR induced photodissociation

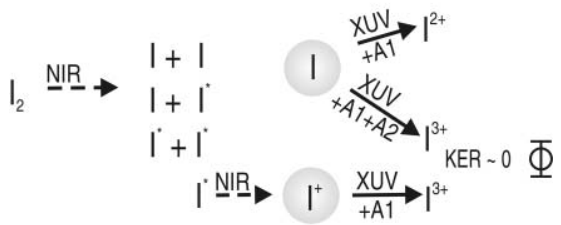

Process B: XUV driven nuclear dynamics

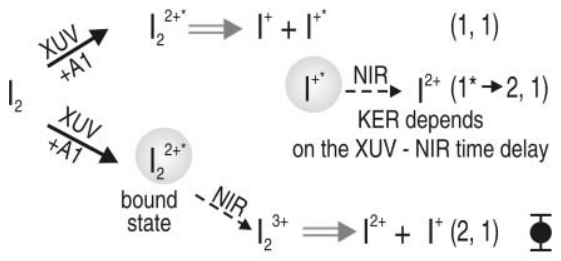

Process C: XUV + NIR driven electron dynamics

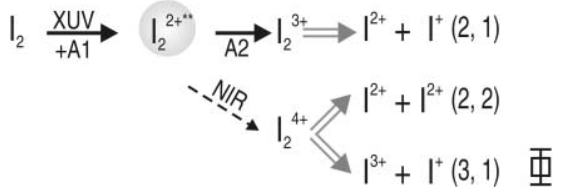

FIG. 3. $\mathrm{I}_{2}$ photofragmentation processes $\mathrm{A}, \mathrm{B}$, and $\mathrm{C}$ as discussed in Secs. III A, III B, and III C, respectively. The probed molecular or atomic state is highlighted in gray. Symbols on the right side correspond to the transient profiles plotted in Fig. 4.

analysis reveals several transient channels driven by different processes $\mathrm{A}, \mathrm{B}$, and $\mathrm{C}$ as summarized in Fig. 3. In Sec. III A the atomic signal, which originates from the NIRinduced photodissociation and postdissociatively accessed accessed by the XUV field (process A), is discussed. Section III $\mathrm{B}$ discusses internuclear dynamics governed by Coulomb explosion after XUV core-ionization and subsequent electronic relaxation (process B). In Sec. III C electron dynamics driven by the collective action of XUV and NIR fields within temporal overlap are discussed (process $\mathrm{C}$ ).

Importantly, in molecular iodine the photofragmentation rate governed by Coulomb repulsion is considerably lower than the Auger decay rate. In the case of $\mathrm{I}_{2}^{2+}$, for example, it takes 151 fs (Ref. 17) for $\mathrm{I}^{+}$ions to double the internuclear separation whereas the $4 d^{-1}$ lifetime $^{24}$ is only a few femtoseconds. This means that the $4 d$ vacancy created by the XUV radiation in $I_{2}$ is refilled via $\mathrm{A} 1$ or $\mathrm{A} 1+\mathrm{A} 2$ Auger decay transitions while the iodine ions are almost at rest. Thus, the electron relaxation dynamics is separated in time from the nuclear dynamics initiated by Coulomb explosion and each $(p, q)$ channel is a signature of the corresponding molecular cationic state.

\section{A. NIR-induced photodissociation probed by the XUV pulse}

As discussed in Sec. II the atomic I, I*, as well as ionic $\left(\mathrm{I}^{+}\right)$fragments with low KER are created by the NIR pulse. For a long negative time delay (NIR pulse first) these fragments can be further ionized by the XUV pulse. The $4 d^{-1}$ vacancy in an atomic I fragment will decay via A1 to $\mathrm{I}^{2+}$

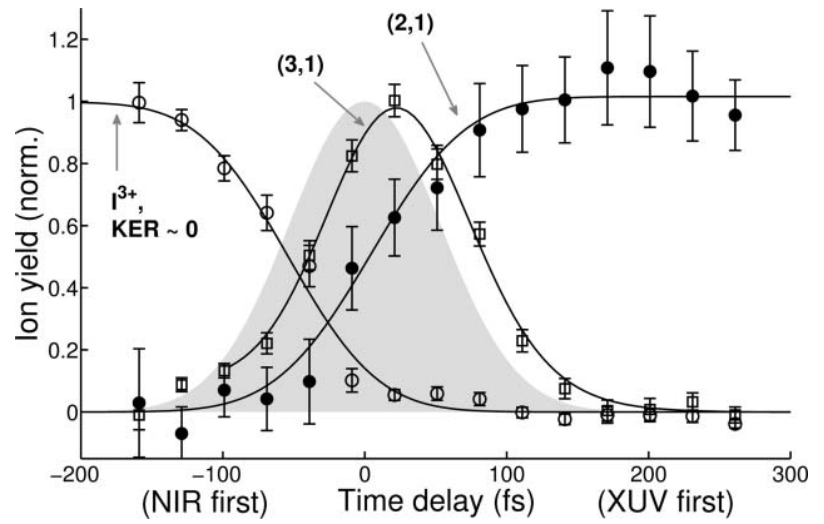

FIG. 4. The transient ion yield as a function of the time delay and corresponding fits to the data (black solid lines). The temporal overlap between XUV and NIR pulses with a Gaussian width of $\sigma=53 \mathrm{fs}$ is depicted by the gray filled curve.

and via $\mathrm{A} 1+\mathrm{A} 2$ to $\mathrm{I}^{3+}$ ions. $^{25}$ In singly charged ions the $\mathrm{A} 1$ decay is preferred ${ }^{26}$ and will lead to the production of $\mathrm{I}^{3+}$. Thus, $\mathrm{I}^{2+}$ and $\mathrm{I}^{3+}$ ionic fragments appearing at negative time delays with KER close to zero arise from the NIR-induced and XUV-probed photodissociation as summarized in Fig. 3 (process A).

The transient $\mathrm{I}^{2+}$ ion signal with low KER at negative time delays can hardly be resolved because of a strong background of low KER ions produced by the XUV radiation [Fig. 1(b)]. On the contrary, the $\mathrm{I}^{3+}$ ions are not created solely by the NIR or XUV field and hence the signal in the $\mathrm{I}^{3+}$ data is almost background free. Figure $2\left(\mathrm{I}^{3+}\right.$ plot) shows a pronounced feature at negative time delays near zero KER (black dash-dotted line) associated with the discussed process A.

The transient $\mathrm{I}^{3+}$ ion yield integrated within the zero KER region as a function of time delay is plotted in Fig. 4 (open circles). The temporal profile was fitted with a smoothed step function (solid line). The shift of the inflection point toward negative time delays (NIR pulse first) with respect to the zero time delay (see Sec. III B for the zero time delay calibration $)$ is $\tau_{\text {diss }}=(55 \pm 10)$ fs within $95 \%$ confidence bounds. Since the ionic charge-state signal does not carry any information about its energetic origin, the deduced effective $\tau_{\text {diss }}$ may represent an average over several initial dissociative states. Another important issue is that the detected $\mathrm{I}^{3+}$ fragments are produced via A1 or A1+A2 Auger decay (process A in Fig. 3). A1 is considered to be extremely fast [about several femtoseconds (Ref. 24)] and hardly affects the extracted $\tau_{\text {diss }}$. The time constant determined for the A2 decay in $\mathrm{I}_{2} \tau_{\mathrm{A} 2}=(23 \pm 11)$ fs (discussed in Sec. III C), however, causes a shift of the inflection point toward longer negative time delays leading to an overestimation of $\tau_{\text {diss }}$. Since the branching ratio of $\mathrm{I}^{3+}$ final states populated via $\mathrm{A} 1$ and $\mathrm{A} 1+\mathrm{A} 2$ remains unknown, the dissociation time is probably shorter than extracted in the current study.

\section{B. Tracing nuclear dynamics triggered by XUV core-ionization}

At positive time delays $\left(\mathrm{I}^{2+}\right.$ plot in Fig. 2) the intensity in the $(2,1)$ channel is substantially increased. The transient 
ion yield in this decay channel is plotted in Fig. 4 (closed circles) and is indicative of a $\mathrm{I}_{2}^{2+*}$ transient molecular cationic state populated by A1 and further ionized by the NIR pulse to $\mathrm{I}_{2}^{3+}$ (process B in Fig. 3). As the signal stays at the same level for positive delays (of several picoseconds, data not shown), $\mathrm{I}_{2}^{2+*}$ has to be a bound state. The loosely bound Rydberg electron, however, makes it susceptible to postionization with a moderate NIR field. The transient profile was fitted with a smoothed step function and the inflection point $\mathrm{t}_{0} \pm 8$ fs (determined within $95 \%$ confidence bounds) was used to determine the maximum overlap between XUV and NIR pulses, i.e., the zero time delay. This definition is justified because on the time scale of our experiment the time constant for the A1 decay, $\tau_{\mathrm{Al}}$, is very small ${ }^{24}$ and, thus, the $\mathrm{I}_{2}^{2+*}$ state can be considered to be instantaneously populated by A1.

An additional feature appears as a shoulder at the lower KER side of the pronounced $(2,1)$ signal. It indicates that some fraction of $\mathrm{I}_{2}^{2+*}$ is also created on a repulsive potential and consequently undergoes Coulomb explosion into $\mathrm{I}^{+}+\mathrm{I}^{+*}$. The KER in this case depends on the internuclear separation of $\mathrm{I}^{+}$and $\mathrm{I}^{+*}$ fragment ions at the instant of the postionization by the NIR pulse and, thus, on the time delay between XUV and NIR pulses (as summarized in Fig. 3, process B). To indicate the dependence of the KER on the XUV-NIR time delay, the corresponding decay channel is referred to as $\left(1^{*} \rightarrow 2,1\right)$. This transient feature provides a possibility to trace the Coulomb explosion of the molecular dication in time but strongly overlaps with the $(2,1)$ channel. In order to isolate the $\left(1^{*} \rightarrow 2,1\right)$ contribution, the following procedure was applied to the data set of Fig. 2: first, an ion trace at a long negative time delay was taken as a reference. Secondly, this reference trace-scaled to the corresponding ion yield within the $(2,1)$ decay channel - was subtracted from the ion trace at each time delay. The resulting $\mathrm{I}^{2+}$ ion yield difference spectra at selected time delays (solid lines) are plotted in Fig. 5(a). The shift of the center of mass of the transient feature attributed to the $\left(1^{*} \rightarrow 2,1\right)$ channel from the TOF corresponding to the $(2,1)$ repulsion toward lower KER is noticeable at positive time delays (NIR after XUV).

The center of mass shift extracted from Fig. 5(a) was recalculated into a corresponding KER, which on this part is a measure of the internuclear separation $R$. Figure $5(\mathrm{~b})$ shows the evolution of the deduced internuclear distance $R$ (normalized to the equilibrium $R_{e}=2.66 \AA$ ) as a function of the time delay between XUV and NIR pulses. Both, $\mathrm{I}^{2+}$ ions with the initial momentum toward (filled circles) and backward (open circles) with respect to the detector, were analyzed. In the proposed model [see inset in Fig. 5(b)] the XUV pump pulse excites the molecular wave packet to the repulsive $\mathrm{I}^{+}+\mathrm{I}^{+*}$ state. The subsequent NIR-induced liberation of the loosely bound Rydberg electron $\mathrm{I}^{+*} \rightarrow \mathrm{I}^{2+}$ introduces an additional gain in kinetic energy, and, thus, causes a projection of the molecular wave packet onto the higher $\mathrm{I}^{2+}+\mathrm{I}^{+}$ Coulomb state. Hence, the temporally delayed NIR pulse probes the time-dependent position $R(t)$ of the wave packet in the $\mathrm{I}^{+}+\mathrm{I}^{+*}$ Coulomb potential. The temporal evolution of internuclear separation can be modeled by considering the Coulomb repulsion between two point charges and integrating the classical equations of motions similar as in Ref. 17. The
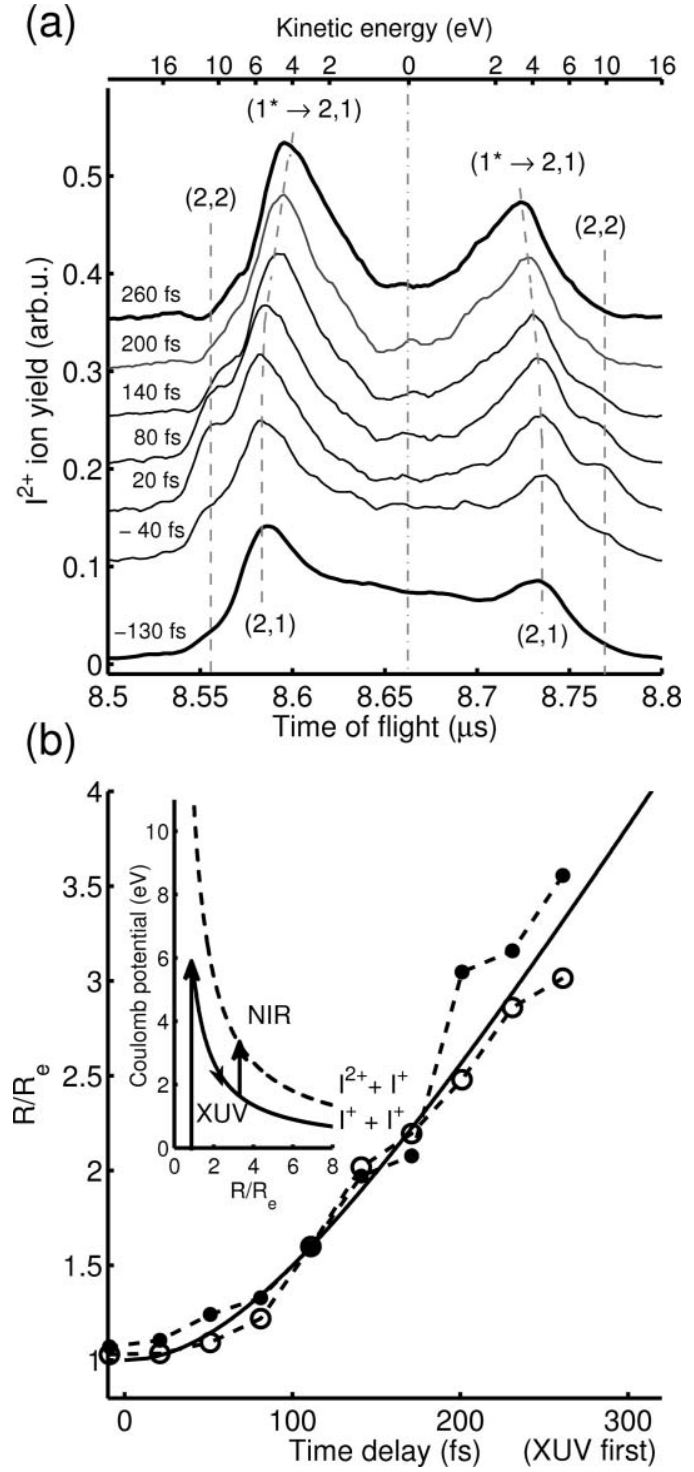

FIG. 5. $\mathrm{I}^{2+}$ ion yield difference spectra at certain time delays (solid lines). The data were extracted from Fig. $2\left(\mathrm{I}^{2+}\right.$ plot) after background subtraction as explained in the text. The fragment's TOF are indicated by vertical dashed lines. (b) The temporal evolution of the internuclear distance $R$ normalized to $R_{e}$, as deduced from the center-of-mass shift of the transient feature shown in (a). The inset shows a simplified scheme for probing the evolution of the $\left(1^{*} \rightarrow 2,1\right)$ channel. The two data sets represent $\mathrm{I}^{2+}$ fragments with the initial momentum directed toward (filled circles) and away (open circles) from the detector. The solid line represents the calculation based on the two-pointcharge model.

solid line in Fig. 5(b) represents the calculation of the time dependent increase of the distance between two recoiling $\mathrm{I}^{+}$ point-charges.

The motion of molecular wave packets on repulsive potentials has so far been studied by using strong laser excitation. ${ }^{27,28}$ However, the application of optical-optical pump-probe schemes to molecules with a large number of valence electrons such as $\mathrm{I}_{2}$ is difficult at short time delays (i.e., at $R_{e}<R<2 R_{e}$ ) because the contributions of the pump and probe fields can hardly be separated. Moreover, the intensity modulation caused by the interference of the pump and probe fields of the same spectrum, polarization, and 
propagation direction requires an implementation of phaseshift techniques. ${ }^{29}$

\section{Electron dynamics driven by the collective action of XUV and NIR fields}

Within the XUV-NIR temporal overlap the $(1,1)$ and $(1,2)$ feature is bleached $\left(\mathrm{I}^{+}\right.$plot in Fig. 2). Instead, two decay channels with symmetric $(2,2)$ and asymmetric $(3,1)$ charge distribution $\left(\mathrm{I}^{2+}\right.$ and $\mathrm{I}^{3+}$ plots, respectively) appear. Both pathways originate from the explosion of the $\mathrm{I}_{2}^{4+}$ molecular cation. A precursor for $\mathrm{I}_{2}^{4+}$ is most probably the doubly excited $\mathrm{I}_{2}^{2+* *}$ molecular dication which decays to $\mathrm{I}_{2}^{3+}$ via A2 (process $\mathrm{C}$ in Fig. 3). Such double excitations can be expected ${ }^{13}$ in $\mathrm{I}_{2}$ and similar effects were observed in related studies on Xe. ${ }^{4}$

The pronounced signal from the biased $(3,1)$ decay channel is an unexpected finding because it was shown in photoion-photoion coincidence measurements ${ }^{15}$ that the highly charged molecular cationic states $\mathrm{I}_{2}^{4+}$ and $\mathrm{I}_{2}^{5+}$ decay preferably via channels with symmetric charge distribution $\mathrm{I}^{2+}+\mathrm{I}^{2+}$ and $\mathrm{I}^{2+}+\mathrm{I}^{3+}$, respectively. An asymmetric charge distribution was found to be inefficient. ${ }^{15}$ The plausible explanation for the charge-asymmetric fragmentation pathway is that the electric field of the NIR probe pulse polarizes a molecular ion by exciting a charge-transfer state. Charge-transfer transitions have already been shown to exist in halogen molecules. ${ }^{30}{ }^{2} \mathrm{I}_{2}$ the D-band system in the absorption spectra $\left(\sigma_{g} 5 p \rightarrow \sigma_{u} 5 p\right)$ corresponds to such a type of transition. ${ }^{30}$

Charge-transfer states are known to be parallel-type transitions, i.e., an electron is transferred between two atoms along the molecular axis. The molecular gas target in the current study is not aligned. The charge-transfer transition can thus be excited only for molecules with their axis occasionally oriented along the polarization of the NIR field. Consequently, if the photofragmentation is initiated from a chargetransfer state, the fragment distribution will be anisotropic, maximal in the direction of the NIR field polarization and suppressed in the perpendicular direction. Since our TOF spectrometer has an angle-selecting aperture, only fragments with an initial momentum within the $\sim 15^{\circ}$ acceptance angle can reach the detector. As shown in Fig. 6, the anisotropy in the $(3,1)$ channel is indeed observed. The $\mathrm{I}^{3+}$ ion yield is considerably lower in the case of the perpendicular orientation of the NIR field polarization with respect to the axis of the TOF spectrometer (gray dashed line) compared to the parallel (black solid line) orientation. Both traces were measured under otherwise the same experimental conditions. The ion yield within other $(p, q)$ channels is not influenced by the polarization of the NIR field (data not shown).

The temporal profiles of both, $(2,2)$ and $(3,1)$ photofragmentation pathways are found to be nearly the same. Figure 4 displays the transient $(3,1)$ ion yield as a function of the time delay between XUV and NIR pulses. The temporal profile is fitted by the convolution of the instrument response function (Gaussian with width $\sigma=(53 \pm 10)$ fs) with an exponential decay $\tau_{\mathrm{A} 2}$. Since the life time of the intermediate $\mathrm{I}_{2}^{2+* *}$ state is considerably shorter than the current temporal

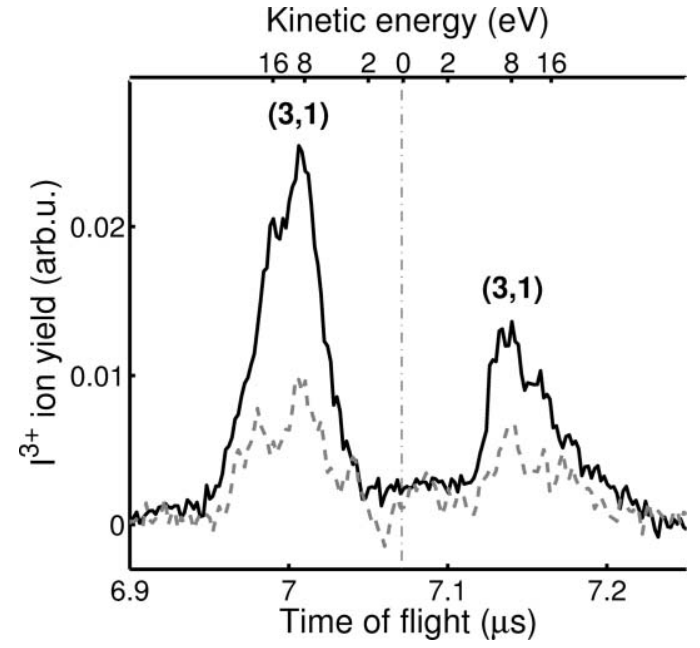

FIG. 6. Momentum resolved $\mathrm{I}^{3+}$ ion yield with parallel (black solid line) and perpendicular (gray dashed line) polarization of the NIR field with respect to the axis of the ion spectrometer measured under otherwise the same experimental conditions. Each curve is an average of about 80 single traces. A background trace taken at a long positive time delay (XUV first) was subtracted in both cases. The scale at the top indicates the fragment kinetic energy (half of KER) for ions with the initial momentum on the spectrometer's axis.

resolution, $\tau_{\mathrm{A} 2}$ cannot be extracted precisely from the direct fit to the data. However, as discussed previously in time-resolved studies of several molecular systems, ${ }^{31}$ the shift of the signal maximum with respect to the zero time delay is sensitive to the decay time. Thus, the shift of the $(3,1)$ signal (Fig. 4, open squares) with respect to the XUV-NIR temporal overlap (gray filled area) provides a measure of $\tau_{\mathrm{A} 2}=(23 \pm 11)$ fs. This is compatible with $\tau_{\mathrm{A} 2}=(30.8 \pm 1.4)$ fs deduced for Xe. ${ }^{4}$

\section{SUMMARY AND CONCLUSION}

Excitation of $\mathrm{I}_{2}$ with timed intense NIR and XUV pulses reveals rich intertwined electronic and nuclear dynamics. The greatly different light frequencies drive different physical mechanisms. Measurement of the kinetic energy release of the recoiling fragments allowed us to identify-in the same experimental run-several relaxation and fragmentation pathways, such as molecular Auger decay, Coulomb explosion of highly charged molecular ionic states, as well as fragmentation from dissociative states. In the following, we summarize the three most prominent findings.

Direct excitation into dissociative states via NIR multiphoton absorption favors fragmentation into neutral atoms with correspondingly small KER. The deduced characteristic dissociation time constant of $(55 \pm 10)$ fs is in a range of photodissociation times of $\sim 40$ fs (Refs. 1 and 2 ) and $(55 \pm 15)$ fs (Ref. 3) reported for $\mathrm{Br}_{2}$ in time-resolved photoelectron spectroscopy studies. The electronic structure of the free $\mathrm{Br}$ atom, however, seems to be established $\sim 85$ fs after excitation. ${ }^{3}$ Hence, care should be taken when comparing time constants deduced with different techniques because the detected physical observables may be subject to different explicit evolutions. This is also supported by a recently introduced method, ${ }^{6}$ where the time-dependent high-harmonic yield from $\mathrm{Br}_{2}$ suggests that $\mathrm{Br}$ atoms (or strictly speaking the atomic character 
of the electronic wavefunction) are formed only after $300 \mathrm{fs}$. For the case studied in this work, the deduced value refers to the time after NIR excitation when the internuclear distance suppresses further exchange of electrons between the atomic partners.

At positive time delays, XUV excitation precedes the NIR pulse and predominantly creates $I 4 d^{-1}$ hole states with subsequent rapid Auger decay. We found evidence for the formation of a long-lived excited $\mathrm{I}_{2}^{2+*}$ state, where the probing NIR field removes the excited electron and the triply charged molecule experiences rapid Coulomb explosion. Another fraction of the $\mathrm{I}_{2}^{2+*}$ population directly undergoes Coulomb explosion, leaving one of the atomic fragments in an excited I ${ }^{+*}$ state. Upon removal of the loosely bound Rydberg electron by the NIR pulse, an $\mathrm{I}^{2+}$ ion is formed. The delaydependent KER of this fragment maps the movement of the molecular wave packet on the repulsive Coulomb potential starting from the equilibrium internuclear distance.

The XUV induced Auger decay also populates an $\mathrm{I}_{2}^{2+* *}$ short-lived doubly excited state, which spontaneously decays to $\mathrm{I}_{2}^{3+}$ in a second Auger transition with a revealed characteristic time constant $\tau_{\mathrm{A} 2}=(23 \pm 11)$ fs. Within the temporal overlap this intermediate state can also be ionized to $\mathrm{I}_{2}^{4+}$ by the NIR field, initiating charge-symmetric as well as charge-asymmetric photofragmentation. The observation of the charge-asymmetric $\mathrm{I}_{2}^{4+} \rightarrow \mathrm{I}^{3+}+\mathrm{I}^{+}$fragmentation channel is indicative for the excitation of a charge-transfer by the NIR field. So far, the charge-asymmetric dissociation was observed for MEDI of molecules ${ }^{32}$ in an order of magnitude more intense $\left(>10^{14} \mathrm{~W} / \mathrm{cm}^{2}\right)$ and shorter $(<100 \mathrm{fs})$ laser pulses than used in the current study.

The spectral information in delay-dependent recoil momentum spectrometry helps disentangling multiple electronic and nuclear dynamical processes driven by the collective action of XUV and NIR pulses. However, it does not deliver a spectroscopic fingerprint of an intermediate state, as provided by photoelectron spectroscopy. In the present work, we have used analogies to previous transient charge state studies in Xe atoms ${ }^{4}$ which share almost the complete electronic shell with I and whose electronic structure, including excited Rydberg states, ${ }^{33,34}$ is relatively well understood. Future time-resolved studies shall combine the capabilities of electron and ion detection in order to obtain the most comprehensive view at the complex dynamics considered here.

\section{ACKNOWLEDGMENTS}

This work was supported by the Bundesministerium für Bildung und Forschung (BMBF 05-KS4PB1/0 within FSP 301). Professor Nikolay M. Kabachnik is gratefully acknowledged for fruitful discussions and his continuing interest in this work. The authors would like to thank the scientific and technical team of FLASH for machine operation and support at the beamline making this work possible.

${ }^{1}$ L. Nugent-Glandorf, M. Scheer, D. Samuels, A. Mulhisen, E. Grant, X. Yang, V. Bierbaum, and S. Leone, Phys. Rev. Lett. 87, 193002 (2001).
${ }^{2}$ L. Nugent-Glandorf, M. Scheer, D. Samuels, V. Bierbaum, and S. Leone, J. Chem. Phys. 117, 6108 (2002).

${ }^{3}$ P. Wernet, M. Odelius, K. Godehusen, J. Gaudin, O. Schwarzkopf, and W. Eberhardt, Phys. Rev. Lett. 103, 013001 (2009).

${ }^{4}$ M. Uiberacker, T. Uphues, M. Schultze, A. J. Verhoef, V. Yakovlev, M. F. Kling, J. Rauschenberger, N. M. Kabachnik, H. Schroeder, M. Lezius, K. L. Kompa, H. G. Muller, M. J. J. Vrakking, S. Hendel, U. Kleineberg, U. Heinzmann, M. Drescher, and F. Krausz, Nature (London) 446, 627 (2007)

${ }^{5}$ T. Uphues, M. Schultze, M. F. Kling, M. Uiberacker, S. Hendel, U. Heinzmann, N. M. Kabachnik, and M. Drescher, New J. Phys. 10, 025009 (2008).

${ }^{6}$ H. J. Wörner, J. B. Bertrand, D. V. Kartashov, P. B. Corkum, and D. M. Villeneuve, Nature (London) 466, 604 (2010).

${ }^{7}$ P. Morin and I. Nenner, Phys. Scr. T17, 171 (1987).

${ }^{8}$ P. Morin and I. Nenner, Phys. Rev. Lett. 56, 1913 (1986).

${ }^{9}$ S. Cunovic, N. Muller, R. Kalms, M. Krikunova, M. Wieland, M. Drescher, T. Maltezopoulos, U. Fruhling, H. Redlin, E. Plonjes-Palm, and J. Feldhaus, Appl. Phys. Lett. 90, 121112 (2007).

${ }^{10}$ M. Wellhöfer, M. Martins, W. Wurth, A. A. Sorokin, and M. Richter, J. Opt. A, Pure Appl. Opt. 9, 749 (2007).

${ }^{11}$ P. Radcliffe, S. Duesterer, A. Azima, H. Redlin, J. Feldhaus, J. Dardis, K. Kavanagh, H. Luna, J. P. Gutierrez, P. Yeates, E. T. Kennedy, and J. T. Costello, Appl. Phys. Lett. 90, 131108 (2007).

${ }^{12}$ K. Tiedtke, A. Azima, N. von Bargen, L. Bittner, S. Bonfigt, S. Duesterer, B. Faatz, U. Fruehling, M. Gensch, C. Gerth, N. Guerassimova, U. Hahn, T. Hans, M. Hesse, K. Honkavaar, U. Jastrow, P. Juranic, S. Kapitzki, B. Keitel, T. Kracht, M. Kuhlmann, W. B. Li, M. Martins, T. Nunez, E. Ploenjes, H. Redlin, E. L. Saldin, E. A. Schneidmiller, J. R. Schneider, S. Schreiber, N. Stojanovic, F. Tavella, S. Toleikis, R. Treusch, H. Weigelt, M. Wellhoefer, H. Wabnitz, M. V. Yurkov, and J. Feldhaus, New J. Phys. 11, 023029 (2009).

${ }^{13}$ F. Comes, U. Nielsen, and W. Schwarz, J. Chem. Phys. 58, 2230 (1973).

${ }^{14}$ U. Becker, D. Szostak, H. Kerkhoff, M. Kupsch, B. Langer, R. Wehlitz, A. Yagishita, and T. Hayaishi, Phys. Rev. A 39, 3902 (1989).

${ }^{15}$ B. Boo and N. Saito, J. Electron Spectrosc. Relat. Phenom. 127, 139 (2002).

${ }^{16}$ U. Fruehling, M. Wieland, M. Gensch, T. Gebert, B. Schuette, M. Krikunova, R. Kalms, F. Budzyn, O. Grimm, J. Rossbach, E. Ploenjes, and M. Drescher, Nat. Photonics 3, 523 (2009).

${ }^{17}$ P. Dietrich, D. Strickland, and P. Corkum, J. Phys. B 26, 2323 (1993).

${ }^{18}$ J. H. Posthumus, A. J. Giles, M. R. Thompson, and K. Codling, J. Phys. B 29, 5811 (1996).

${ }^{19}$ J. Tremblay, M. Larzilliere, F. Combet-Farnoux, and P. Morin, Phys. Rev. A 38, 3804 (1988).

${ }^{20}$ R. Mulliken, J. Chem. Phys. 55, 288 (1971).

${ }^{21}$ M. Drescher, U. Fruehling, M. Krikunova, T. Maltezopoulos, and M. Wieland, J. Phys. B 43, 194010 (2010).

${ }^{22}$ T. Maltezopoulos, S. Cunovic, M. Wieland, M. Beye, A. Azima, H. Redlin, M. Krikunova, R. Kalms, U. Fruehling, F. Budzyn, W. Wurth, A. Foehlisch, and M. Drescher, New J. Phys. 10, 033026 (2008).

${ }^{23}$ M. Krikunova, T. Maltezopoulos, A. Azima, M. Schlie, U. Fruehling, H. Redlin, R. Kalms, S. Cunovic, N. M. Kabachnik, M. Wieland, and M. Drescher, New J. Phys. 11, 123019 (2009).

${ }^{24}$ J. Cutler, G. Bancroft, and K. Tan, J. Chem. Phys. 97, 7932 (1992).

${ }^{25}$ L. Nahon, A. Svensson, and P. Morin, Phys. Rev. A 43, 2328 (1991).

${ }^{26}$ H. Kjeldsen, P. Andersen, F. Folkmann, H. Knudsen, B. Kristensen, J. West, and T. Andersen, Phys. Rev. A 62, 020702 (2000).

${ }^{27}$ J. Larsen, N. Morkbak, J. Olesen, N. Bjerre, M. Machholm, S. Keiding, and H. Stapelfeldt, J. Chem. Phys. 109, 8857 (1998).

${ }^{28}$ H. Stapelfeldt, E. Constant, H. Sakai, and P. Corkum, Phys. Rev. A 58, 426 (1998).

${ }^{29}$ G. Gibson, R. Coffee, and L. Fang, Phys. Rev. A 73, 023418 (2006).

${ }^{30}$ R. Mulliken, J. Chem. Phys. 7, 20 (1939).

${ }^{31}$ P. Farmanara, O. Steinkellner, M. Wick, M. Wittmann, G. Korn, V. Stert, and W. Radloff, J. Chem. Phys. 111, 6264 (1999).

${ }^{32}$ G. Gibson, M. Li, C. Guo, and J. Nibarger, Phys. Rev. A 58, 4723 (1998).

${ }^{33}$ H. T. Wang, W. S. Felps, G. L. Findley, A. R. P. Rau, and S. P. McGlynn, J. Chem. Phys. 67, 3940 (1977).

${ }^{34}$ J. A. Dagata, G. L. Findley, S. P. McGlynn, J. P. Connerade, and M. A. Baig, Phys. Rev. A 24, 2485 (1981). 